\section{IDDF2019-ABS-0305 PSOAS MUSCLE THICKNESS AS MEASURE FOR SARCOPENIA AND PROGNOSIS IN LIVER CIRRHOSIS}

Randall Isaac Tan*, Ira Inductivo-Yu, Darlene Jumawan. National Kidney and Transplant Institute, Philippines

\subsection{6/gutjnl-2019-IDDFabstracts.305}

Background Malnutrition is an overlooked complication of liver cirrhosis. Sarcopenia, a marker of malnutrition, is an independent prognostic factor for mortality in liver cirrhosis.

We aimed to evaluate psoas muscle thickness divided by height (PMTH) as a measure for the degree of sarcopenia and determine its correlation with the clinical outcomes.

Methods Sixty-five liver cirrhosis patients with available abdominal CT scan were reviewed in this retrospective cohort study. PMTH were measured and correlated with clinical parameters and outcomes. The diagnostic performance for predicting long-term mortality of PMTH, Child-Pugh, MELD and MELD-Na score was determined by calculating the area under the receiver-operating curve (AUROC) and cut-off values were derived. Event rate of specific complications was analyzed using the cut-off. Kaplan Meier with a log-rank test was used to compare survival rates in the low PMTH vs. high PMTH group. Cox regression analysis was used to determine the variables predictive of mortality.

Results There was no significant difference among the PMTH values between cirrhotic patients without HCCA and with HCCA $(p=0.652)$. BMI showed a significant correlation to PMTH with $\mathrm{p}=0.001 \quad(\mathrm{r}=0.415)$. A weak negative correlation was noted between liver-related complications and PMTH. The best cut-off value of PMTH to predict 6-month mortality was derived at $13.45 \mathrm{~mm} / \mathrm{m}$. Low PMTH was significantly predictive of the occurrence of SBP with $p=0.032$. The performance of $\mathrm{PMTH}$ in 6-month mortality was not significant $(p=0.517)$. Kaplan Meier graph revealed that high PMTH group had a higher survival rate (figure 1). The univariate Cox-regression analysis demonstrated that age and lower serum $\mathrm{Na}$ were significantly associated with mortality $(\mathrm{p}=0.005$ and $\mathrm{p}=0.045)$.

\section{Survival Functions}

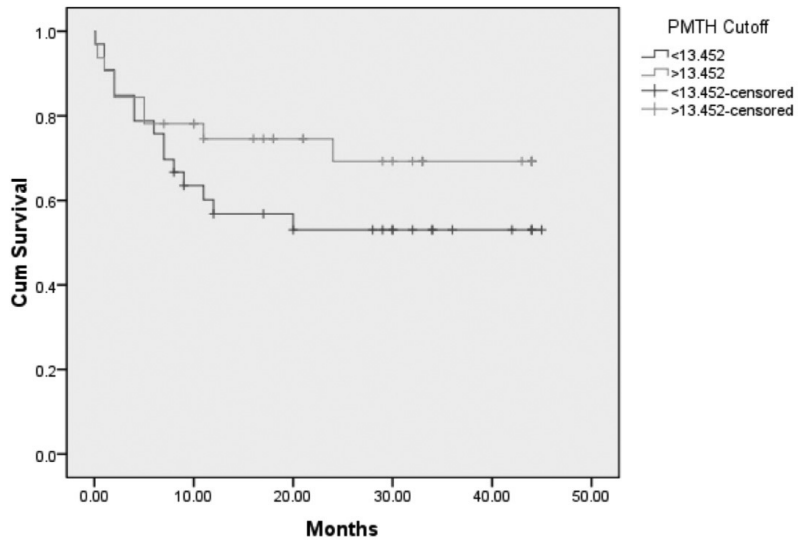

Abstract IDDF2019-ABS-0305 Figure 1 Survival rate among low PMTH and high PMTH groups
Conclusions Psoas muscle thickness index is a promising tool for skeletal muscle assessment among cirrhotic patients. Low psoas muscle thickness is predictive of SBP occurrence. PMTH can be a supplemental prognostic marker on top of the available scoring systems in liver cirrhosis.

\section{IDDF2019-ABS-0306 RISK OF SPONTANEOUS BACTERIAL PERITONITIS WITH USE OF PROTON PUMP INHIBITORS - A SYSTEMIC REVIEW AND META-ANALYSIS}

${ }^{1}$ Marianne Linley Sy*, ${ }^{2}$ Jose Isagani Janairo, ${ }^{1} J u l i e t$ Gopez-Cenvantes, ${ }^{1}$ Ian Homer Cua. 'Institute of Digestive and Liver Diseases, St. Luke's Medical Center - Global City, Philippines; ${ }^{2}$ Biology Department, College of Science, De La Salle University, Philippines

\subsection{6/gutjnl-2019-IDDFabstracts.306}

Background Spontaneous bacterial peritonitis (SBP) is a frequent complication seen among cirrhotic patients. The use of proton pump inhibitors (PPIs) has been suggested to be associated with a higher incidence of SBP. However, previous studies including case controls, cohorts, and meta-analyses provided conflicting conclusions. In light of newer studies that were done to re-evaluate the causality of PPI use and development of SBP, we aim to re-assess the association between PPI use and SBP incidence with larger and better quality data.

Methods Databases of Medline, Cochrane, and Google Scholar were used to search for relevant articles. Two reviewers independently assessed the quality of each included study. Disagreements were resolved by the third author. Preferred Reporting Items for Systematic Reviews and MetaAnalyses (PRISMA) statement was followed. Pooled odds ratios (ORs) with 95\% confidence intervals (CIs) were calculated. Sub-group analyses were done to decrease the heterogeneity.

Results A total of twenty-one studies: ten case-control, ten cohorts, and one randomized controlled trial, involving 13,862 patients were analyzed. The overall results (figure 1) showed a statistically significant association between SBP and PPI use (pooled odds ratio (OR): 2.02, 95\% CI of 1.51 to 2.69). Substantial heterogeneity was observed. On sub-group analysis involving cohort and randomized controlled trial, the association was weaker (OR: 1.36 with 95\% CI of 1.17 to $1.57 \mathrm{p}<0.0001)$ but still statistically significant and with minimal heterogeneity $\left(\mathrm{Chi}^{2} \mathrm{p}=0.11 ; \mathrm{I}^{2}=36 \%\right)$. For casecontrol studies, the OR is 2.98 with $95 \%$ CI of 2.07 to 4.27. The funnel plot was asymmetric suggesting publication bias.

Conclusions This meta-analysis sheds light on the conflicting results raised by previous studies regarding the association of SBP with PPI use. The present meta-analysis showed that there is a weak association, although statistically significant, between SBP and PPI use. Thus, this updated meta-analysis suggests judicious use of PPI among cirrhotic patients with ascites. 